\title{
Alterations in Antigen Expression in Superficial Bladder Cancer
}

\author{
H. Barton Grossman, MD1, Raymond W. Washington, Jr., BS1, Thomas E. Carey, PhD2, and Monica \\ Liebert, PhD1 \\ 1 Section of Urology, University of Michigan Medical School, Ann Arbor, Michigan 48109 \\ 2 Department of Surgery and Department of Otorhinolaryngology, University of Michigan Medical School, Ann \\ Arbor, Michigan 48109
}

\begin{abstract}
Bladder cancer can be viewed as a prototype for carcinogen-induced neoplasia. This has been demonstrated experimentally in a variety of systems and in man through epidemiological studies of occupational exposure to putative carcinogens. The natural history of this neoplasm demonstrates recurrence in time and space, i.e., multifocal disease. This clinical scenario is precisely what would be expected if a target tissue, e.g., urothelium, was continuously exposed to a weak carcinogen. The detection of gross disease is clinically easy. However, the ability to intervene at early stages and monitor the success of this treatment requires the definition of early markers for bladder cancer.

Integrins are a family of cell surface proteins, many of which function as receptors for extracellular matrix components. Normal epithelial cells express the integrin $\alpha 6 \beta 4$ in association with an anchoring structure known as the hemidesmosome. Urothelium expresses $\alpha 6 \beta 4$ on the basal layer of cells similar to the distribution seen on other epithelial surfaces. Even early stages of bladder cancer demonstrate an alteration in the expression of this integrin. Low-stage bladdertumors express $\alpha 6 \beta 4$ diffusely throughout the tumor as well as at the invading margin. Altered expression of $\alpha 6 \beta 4$ may be an early marker for bladder cancer which may contribute to an invasive phenotype.

A second potential marker is detected by DD23, an lgG1 murine monoclonal antibody triggered by the immunization of a $\mathrm{BALB} / \mathrm{C}$ mouse with a fresh human bladder tumor specimen. The antigen detected by DD23 is not present on normal urothelial specimens. It is expressed on $81 \%$ of bladder tumors tested and is present on both low-grade, non-invasive and high-grade, invasive tumors. Although no normal bladder or ureteral tissues were DD23-positive, two of five histologically normal bladder areas from patients who had radical cystectomies for bladder cancer expressed the DD23 antigen. The significance of this finding has not yet been completely evaluated; however, it may represent an early neoplastic change prior to obvious histologic abnormality.

Antigens associated with bladder cancer may be helpful in the early detection of bladder cancer and in providing markers useful in future chemoprevention trials. (c) 1992 Wiley-Liss, inc.
\end{abstract}

Key words: bladder cancer, chemoprevention, integrin, monoclonal antibody, tumor-associated antigen

Both clinical and experimental evidence suggest that at least a proportion of biologically and clinically relevant bladder cancer is a result of carcinogen-induced neoplasia [1,2]. Bladder cancer is commonly associated with multifocal recurrences in time and space, lending further clinical support to the hypothesis that fieldchanges are common with this neoplasm. The clinical detection of gross bladder cancer is readily accomplished with various clinical tools

Supported by grant PDT-409 from the American Cancer Society and grant CA56973 from the National Cancer Institute.

다 1992 Wiley-Liss, Inc. including cytology, flow cytometry, cystoscopy, and a variety of imaging techniques. However, the detection of sub-clinical changes has proved to be a significant challenge.

Cancer is manifested as alterations in the morphology and behavior of normal cells. At a sub-microscopic level, these changes are associated with modifications in cellular proteins and lipids. Some of these abnormally expressed cellular constituents have been used as tumor markers. One example of this phenomenon is the altered blood group antigen expression associated with bladder cancer cells [3]. We have focused our efforts on evaluating alter- 
ations in antigen expression in bladder cancer to determine how these changes result in the malignant phenotype. Our laboratory has developed monoclonal antibodies to a variety of bladder tumor-associated antigens. Two antigenic determinants are particularly relevant for this discussion and may provide new tools to evaluate early changes in bladder cancer.

The first antigen is the integrin $\alpha 6 \beta 4$. Integrins comprise a family of cell surface proteins, many of which function as receptors for extracellular matrix proteins. We have demonstrated that $\alpha 6 \beta 4$ is expressed differently on malignant urothelium as compared with its normal counterpart. Altered expression of $\alpha 6 \beta 4$ may be an early marker for bladder cancer and may contribute to an invasive phenotype.

The second antigen is defined by the monoclonal antibody DD23. DD23 binds to a partially characterized tumor-associated antigen that is frequently expressed on malignant bladder and breast tissue. DD23 does not bind normal urothelium and may also be an early marker for malignant transformation.

\section{EXPRESSION OF INTEGRIN $\alpha 6 \beta 4$ IN NORMAL AND MALIGNANT UROTHELIUM}

Integrins were evaluated by immunofluorescence or immunoperoxidase staining in frozen sections of normal urothelium and bladder cancer specimens using the monoclonal antibodies BQ16 (anti- $\alpha 6$, IgG1 [4]) and UM-A9 (anti- $\beta 4$, IgG2a [5]). For immunofluorescence, co-staining of identical sections was performed as previously described [6] using isotype-specific secondary antibodies (rhodamine anti-IgG1 and fluorescein isothiocyanate-conjugated antiIgG2a). For immunoperoxidase staining, separate sections of the same tumor were tested with each antibody and the staining pattern was compared.

Normal urothelial cells in ureter or bladder showed highly polarized expression of $\alpha 6 \beta 4$ on the surface of the basal urothelial cells, resulting in a linear staining for antibody at the junction of the basal cells and the lamina propria, as shown in Figure 1. In sections co-stained for both $\alpha 6$ and $\beta 4$ expression, the staining patterns were identical in all cases. This highly polarized expression is similar to the expression

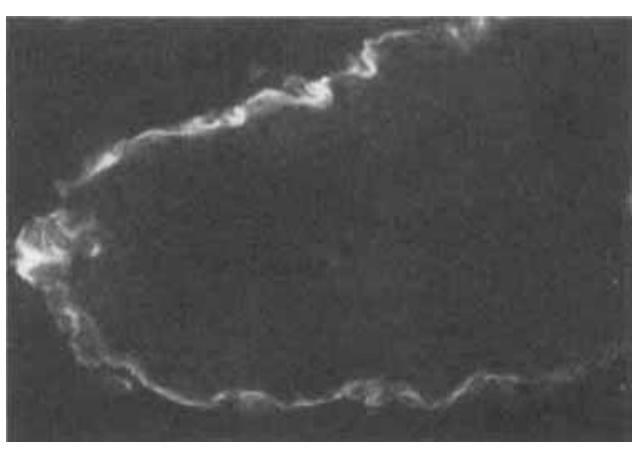

Fig. 1. Immunofluorescence staining of normal ureter with BQ16 shows a linear staining of the surface of the basal urothelial cells. The lumen is on the right side of the photograph. No fluorescence signal was observed in tissues stained with the negative control antibody MOPC-21.

of $\alpha 6 \beta 4$ on normal keratinocytes in the skin [7-12]. Ten low-stage (Ta/T1) bladder cancers tested by either technique showed the normal pattern of staining, with strong expression of both $\alpha 6$ and $\beta 4$ at the junction of the basal cells and the lamina propria. However, $8 / 10$ of these superficial bladder cancer specimens also showed an increased non-polarized expression of both $\alpha 6$ and $\beta 4$ all around the tumor cells, as shown in Figure 2. The staining patterns for $\alpha 6$ and $\beta 4$ were identical.

\section{ANTIBODY DD23 BINDS TO A BLADDER TUMOR-ASSOCIATED ANTIGEN}

DD23 is an IgG1 murine monoclonal antibody, produced after two intraperitoneal immunizations of a mouse with an uncultured polypoid grade III transitional cell carcinoma which was obtained at radical cystectomy. Screening was performed on frozen human tissue sections by immunoperoxidase assay employing the avidin-biotin peroxidase complex technique. The DD23 antigen is not preserved in formalinfixed, paraffin-embedded material. DD23 was selected by immunohistochemical staining of bladder tumors and lack of binding to normal urothelium. Frozen sections evaluated by immunoperoxidase assay showed that DD23 bound to both bladder and breast cancers (Table I). DD23 was expressed more frequently ( $p<0.001$, chi square) on breast cancer $(56 \%)$ than normal breast tissue $(5 \%)$. Both normal and malignant breast tissue were available from 


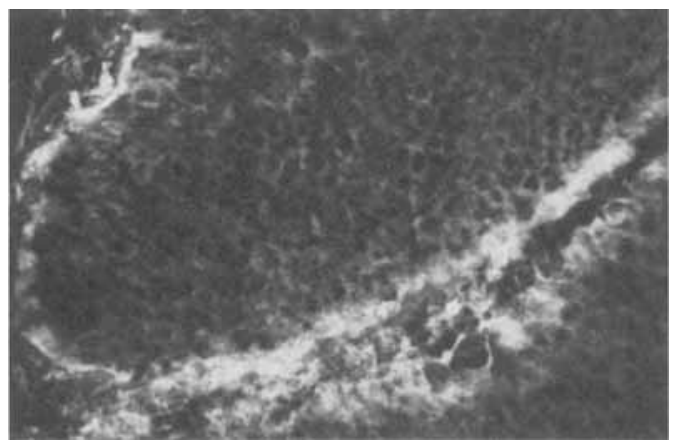

Fig. 2. Immunofluorescence staining of a low-stage bladder cancer with BQ16. Strong staining is seen along the basal surface of the papillary stalk (at bottom) but nonpolarized staining of the tumor cells is also seen. No fluorescence signal was observed in tissues stained with the negative control antibody MOPC-21.

TABLE 1. DD23 Binding to Malignant Tissues

\begin{tabular}{lc}
\hline Tissue & Positive/Total \\
\hline Bladder cancer & $32 / 40$ \\
Breast cancer & $9 / 16$ \\
Renal cancer & $0 / 7$ \\
\hline
\end{tabular}

TABLE II. DD23 Binding to Normal Tissues

\begin{tabular}{lc}
\hline Tissue & Positive/Total \\
\hline Ureter & $0 / 23$ \\
Bladder & $0 / 4$ \\
Breast & $1 / 19$ \\
Kidney & $0 / 7$ \\
Prostate & $0 / 7$ \\
Skin & $0 / 2$ \\
Colon & $0 / 1$ \\
Liver & $0 / 1$ \\
Lung & $0 / 1$ \\
Pancreas & $0 / 1$ \\
Spleen & $0 / 1$ \\
Stomach & $0 / 1$ \\
\hline
\end{tabular}

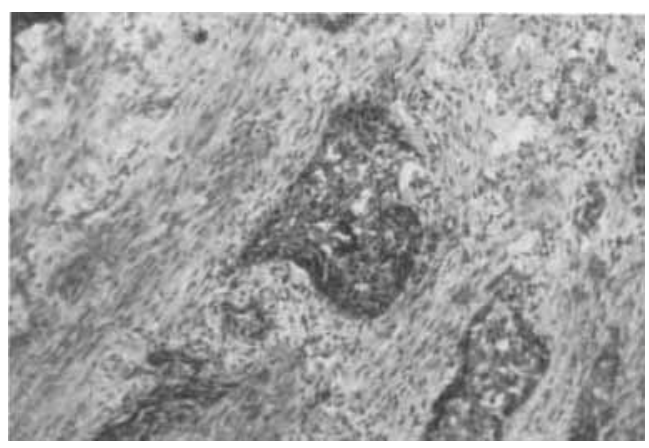

Fig. 3. Immunoperoxidase staining of invasive bladder cancer specimen with DD23. Stain results in a dark brown precipitate around the bladder cancer (in center) invading into the surrounding tissue.

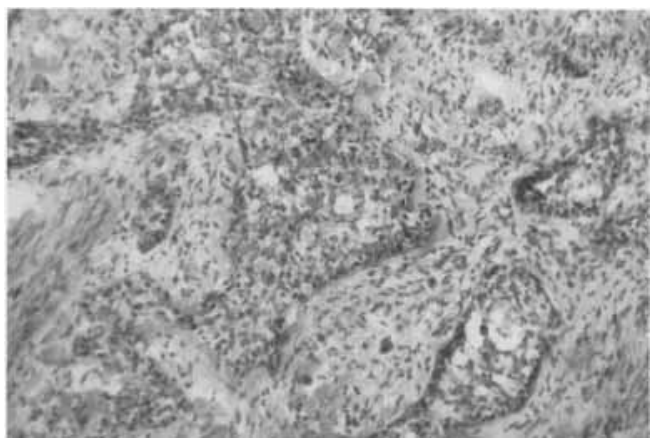

Fig. 4. Control immunoperoxidase staining of invasive bladder cancer specimen (same specimen as Fig. 3) shows the lack of staining of the cancer tissue in the center of the field. This demonstrates the specificity of the staining in Fig. 3.

four individuals. DD23 stained the malignant but not the normal breast tissue in all four cases. DD23 was also not expressed by normal bladder, colon, kidney, liver, lung, pancreas, prostate, skin, spleen, stomach, uterus, or ureter (Table II).

DD23 bound to most (80\%) bladder carcinomas (Figs. 3, 4). The antigen was expressed on both low-grade non-invasive and high-grade invasive tumors. DD23 binds to most invasive bladder cancers in a diffuse fashion; it also stains the limited number of superficial tumors that have been studied. Although no normal bladder or ureteral tissue was DD23-positive, two of five histologically normal bladder areas from patients who had radical cystectomies for bladder cancer expressed the DD23 antigen. 
DD23 binds to only a small proportion of a panel of human bladder cancer cell lines (5/14). UM-UC-13 is a human bladder cancer cell line that expresses the antigen detected by DD23. Extracts of UM-UC-13 were electrophoresed on a $7 \%$ polyacrylamide gel under non-reducing conditions. The proteins on the gel were transferred to nitrocellulose as previously described [13]. Western blots were probed with DD23 and a negative control, monoclonal antibody MOPC21 . The blots were developed with peroxidaseconjugated goat anti-mouse immunoglobulin and 4-chloronaphthol. DD23 binds to a single band with a $\mathrm{Mr} \approx 185,000$.

\section{SUMMARY AND CONCLUSIONS}

Alterations in normal antigen expression regularly accompany loss of differentiation and development of the malignant phenotype. While the functional results of these changes are poorly understood, it is likely that better understanding of their physiologic consequences would result in improved cancer detection, prognosis, and therapy. Both known and unknown proteins may provide sites for such intervention. The integrin $\alpha 6 \beta 4$ is an example of a known protein which is altered in neoplasia and appears to have relevance for bladder cancer. In addition, novel proteins have been found through the application of monoclonal antibodies developed by appropriate immunization and screening techniques. The antigen defined by DD23 appears to belong to this latter category.

Integrins are dimeric cell surface glycoproteins that play important roles in cellular adhesion, wound healing, and differentiation [1416]. Integrin $\alpha 6 \beta 4$, associated with epithelial cells, is expressed in normal skin on the basal layer of keratinocytes. Although $\alpha 6$ can complex with either $\beta 1$ or $\beta 4, \alpha 6$ shows preferential association with $\beta 4$ when $\beta 4$ chains are present [7]. In normal skin, $\alpha 6 \beta 4$ is localized to anchoring structures known as hemidesmosomes [912]. Normal urothelium expresses $\alpha 6 \beta 4$ in a fashion similar to that previously documented in normal skin. Immunofluorescence with monoclonal antibodies to the $\alpha 6$ and $\beta 4$ integrin subunits demonstrate co-localization. In all cases, the distribution of $\alpha 6$ and $\beta 4$ in both normal urothelium and bladder cancer was identical. These results strongly suggest that in both normal and malignant urothelium, $\alpha 6$ is primarily associated with $\beta 4$. Normal urothelium displays the basal distribution of $\alpha 6 \beta 4$ expected for this component of a cellular anchoring structure. In contrast, the distribution of $\alpha 6 \beta 4$ in superficial bladder cancer demonstrates a diffuse cellular distribution in addition to the expected location at the edge of the tumor where it interacts with normal stroma.

Hemidesmosomes play a role in normal cellular attachment to the underlying stroma, so it is therefore likely that aberrant expression of $\alpha 6 \beta 4$ may reflect a functional alteration associated with an invasive phenotype. Altered expression of $\alpha 6 \beta 4$ is a prognostic marker in squamous cell carcinoma of the head and neck [5]. In bladder cancer, the expression of $\alpha 6 \beta 4$ is altered early in the development of neoplasia, i.e., in superficial bladder cancer. We are continuing to evaluate the functional and prognostic significance of this integrin in bladder cancer.

In addition to characterizing the functional effects of alterations in known protein moieties, we have developed monoclonal antibodies to antigens primarily expressed on malignant bladder cells. The production of monoclonal antibodies to tumor-associated antigens is based on the assumption that malignant transformation is associated with detectable changes in the expression of a variety of cellular proteins and lipids. This phenomenon occurs both in vitro and in vivo and has led to a variety of immunization and detection strategies. To avoid selection problems associated with cultured cell lines, we used uncultured human bladder cancer cells for immunization and tumor tissue for screening. This technique worked remarkably well and resulted in antibody DD23 which binds to most bladder tumors even though only $36 \%$ of cultured human bladder cancer cell lines are DD23-positive.

DD23 is not reactive with normal urothelium (ureter and bladder) but binds to an antigen present on most bladder (80\%) and breast (56\%) carcinomas. Despite the lack of DD23 antigen expression on urothelial specimens from normal individuals, two of five patients with bladder cancer expressed this antigen in cystectomy specimens from histologically normal tissues away from the neoplasm. Most patients who require cystectomy manifest multifocal or fieldchange bladder cancer. The significance of DD23 binding to histologically normal areas in 
cystectomy specimens continues to be evaluated by additional histologic characterization of normal and malignant urothelium. However, the data suggest that DD23 may detect changes associated with malignant transformation before conventional microscopy can. DD23 also bound to $5 \%$ of normal breast tissue samples tested; it is not yet known whether this represents early malignant changes or other phenomena.

Antibody DD23 appears to define a new bladder and breast cancer-associated antigen as defined by its molecular weight and as demonstrated by its limited distribution in normal tissues. Additional studies are being performed to determine whether this antibody will provide useful information for the diagnosis and treatment of these diseases.

Both integrin $\alpha 6 \beta 4$ and the antigen defined by DD23 appear to be promising markers for superficial bladder cancer. In addition, a variety of other potentially important markers are also being evaluated, including products of oncogenes and tumor suppressor genes, growth factors and their receptors, proliferation-associated antigens, blood group antigens, and tumorassociated antigens [17]. The interaction and relationship of markers in this arbitrary classification is often complex. For example, the epidermal growth factor receptor (EGFR), a membrane glycoprotein which binds to the epidermal growth factor, is overexpressed in invasive bladder cancers [18]. EGFR is encoded by a gene with strong homology to the proto-oncogene c-erbB2. Although the ligand for c-erbB2 is unknown and its role as a marker for bladder cancer is not as well defined as EGFR, it continues to be evaluated [19]. The blood group antigen Lewis ${ }^{\mathrm{X}}$ is commonly expressed on bladder tumors and may be useful in detecting early disease [3]. Antibody 19A211 also binds to superficial bladder tumors and recognizes a sialoglycoprotein distinct from known blood group antigens [20]. Several monoclonal antibodies have been developed that bind to high molecular weight mucin-like tumor-associated antigens expressed on bladder cancer cells. These include M344 [21] and HMFG1 [22-24]. Numerous other monoclonal antibodies have been developed to well-characterized molecules with known functions and to novel antigens that have yet to be defined.
The clinical tools to detect low-stage bladder cancer are powerful and effective. However, the detection of neoplastic cells prior to the development of gross disease remains an enigma. A variety of probes have been developed to unlock this mystery. Through the analysis of these antigenic systems and the determination of the functional implications of altered antigen expression, early detection and intervention in bladder cancer prior to the development of gross disease may be possible.

\section{REFERENCES}

1. Ward E, Halperin W, Thun M, Grossman HB, Fink B, Koss L, Osorio AM, Schulte P: Bladder tumors in two young males occupationally exposed to MBOCA. Am J Ind Med 14:267-272, 1988.

2. Meigs JW, Marrett LD, Ulrich FU, Flannery JT: Bladder tumor incidence among workers exposed to benzidine: A thirty-year follow-up. J Natl Cancer Inst 76:1-8, 1986 .

3. Sheinfeld J, Reuter VE, Melamed MR, Fair WR, Morse M, Sogani PC, Herr HW, Whitmore WF, Cordon-Cardo C: Enhanced bladder cancer detection with the Lewis $\mathrm{X}$ antigen as a marker of neoplastic transformation. J Urol 143:285-288, 1990.

4. Grossman $\mathrm{HB}$, Liebert M, Flint A, Washington R: DD23: A monoclonal antibody reactive with bladder and breast cancer. Proc AACR 33:312, 1992.

5. Van Waes C, Kozarsky KF, Warren AB, Kidd L, Paugh D, Liebert M, Carey TE: The A9 antigen associated with aggressive human squamous carcinoma is structurally and functionally similar to the newly defined integrin alpha6 beta4. Cancer Res 51:2395-2402, 1991.

6. Liebert M, Lee H-S, Carey TE, Grossman HB: Loss of association of the $\alpha 6 \beta 4$ integrin and collagen VII in invasive bladder cancer. Proc AACR 33:33, 1992.

7. Sonnenberg A, Linders CJT, Daams JH, Kennel SJ: The $\alpha 6 \beta 1$ (VLA-6) and $\alpha 6 \beta 4$ protein complexes: Tissue distribution and biochemical properties. J Cell Sci 96:207-217, 1990.

8. De Luca M, Tamura RN, Kajiji S, Bondanza S, Rossino R, Cancedda R, Marchisio PC, Quaranta V: Polarized integrin mediates human keratinocyte adhesion to basal lamina. Proc Natl Acad Sci USA 87:6888-6892, 1990.

9. Stepp MA, Spurr-Michaud S, Tisdale A, Elwell J, Gipson IK: $\alpha 6 \beta 4$ Integrin heterodimer is a component of hemidesmosomes. Proc Natl Acad Sci USA 87:8970-8974, 1990.

10. Carter WG, Kaur P, Gil SG, Gahr PJ, Wayner EA: Distinct functions for integrins $\alpha 3 \beta 1$ in focal adhesion and $\alpha 6 \beta 4 /$ bullous pemphigoid antigen in a new stable anchoring contact (SAC) of keratinocytes: Relations to hemidesmosomes. J Cell Biol 111:3141-3154, 1990. 
11. Sonnenberg A, Calafat J, Janssen H, Daams H, van der Raaij-Helmer LMH, Facioni R, Kennel SJ, Aplin JD, Baker J, Loizidou M, Gorrod D: Integrin $\alpha 6 \beta 4$ complex is located in hemidesmosomes, suggesting a major role in epithelial cell-basement membrane adhesion. J Cell Biol 113:907-917, 1991.

12. Jones JCR, Kurpakus MA, Cooper HM, Quaranta V: A function for the integrin $\alpha 6 \beta 4$ in the hemidesmosomes. Cell Reg 2:427-438, 1991.

13. Towbin H, Staehelin T, Gordon J: Electrophoretic transfer of proteins from polyacrylamide gels to nitrocellulose sheets: Procedure and some applications. Proc Natl Acad Sci USA 76:4350-4354, 1979.

14. Hertle MD, Adams JC, Watt FM: Integrin expression during human epidermal development in vivo and in vitro. Development 112:193-206, 1991.

15. Hertle MD, Kubler M-D, Leigh IM, Watt FM: Aberrant integrin expression during epidermal wound healing and in psoriatic epidermis. J Clin Invest 89:1892-1901, 1992.

16. Grinnell F: Wound repair, keratinocyte activation and integrin modulation. J Cell Sci 101:1-5, 1992.

17. Fradet Y: Markers of prognosis in superficial bladder cancer. Semin Urol 10:28-38, 1992.

18. Harney JV, Liebert M, Wedemeyer G, Washington R, Stein J, Buchsbaum D, Steplewski Z, Grossman HB: The expression of epidermal growth factor receptor on human bladder cancer: Potential use in radioimmunoscintigraphy. J Urol 146:227-231, 1991.

19. Wright C, Mellon $\mathrm{K}$, Johnston $\mathrm{P}$, Lane DP, Harris AL, Horne CH, Neal DE: Expression of mutant p53, c-erbB2 and the epidermal growth factor re- ceptor in transitional cell carcinoma of the human urinary bladder. Br J Cancer 63:967-970, 1991.

20. Fradet $\mathrm{Y}$, LaRue H, Parent-Vaugeois C, Bergeron A, Dufour C, Boucher L, Bernier L: Monoclonal antibody against a tumor-associated sialoglycoprotein of superficial papillary bladder tumors and cervical condylomas. Int $J$ Cancer 46:990-997, 1990.

21. Fradet Y, Islam N, Boucher L, Parent-Vaugeois C, Tardif M: Polymorphic expression of a human superficial bladder tumor antigen defined by mouse monoclonal antibodies. Proc Natl Acad Sci USA 84:7227-7231, 1987.

22. Burchell J, Durbin $\mathrm{H}$, Taylor-Papadimitriou J Complexity of expression of antigenic determinants, recognized by monoclonal antibodies HMFG-1 and HMFG-2, in normal and malignant human mammary epithelial cells. J Immunol 131:508-513, 1983.

23. Kalofonos HP, Sackier JM, Hatzistylianou M, Pervez S, Taylor-Papadimitriou J, Waxman JH, Lavender JP, Wood C, Epenetos AA: Kinetics, quantitative analysis and radioimmunolocalization using indium-111-HMFG1 monoclonal antibody in patients with breast cancer. Br J Cancer 59:939-942, 1989.

24. Anagnostaki E, Skarlos D, Tamvakis N, Psaropoulou P, Blana E, Bamias A, Legaki S, Aravantinos G, Deliveliotis C, Dimopoulos K: Immunohistochemical and immunocytochemical study of bladder carcinomas using the epithelium-specific, tumour-associated monoclonal antibodies HMFG1 and AUA1. Br J Cancer 10:(Suppl)52-56, 1990. 